\title{
Research on the Teaching of Configuration Design in Engineering Graphics
}

\author{
Chaoyong Guo*, Zhizun Li, Jing Wang \\ Depart. of Vehicle and Electrical Engineering, Shijiazhuang Campus, Army Engineering University of PLA; No.97 \\ Hepingxi Road, Shijiazhuang, Hebei Province, China, 050003 \\ *Corresponding author
}

\begin{abstract}
In engineering design, as the main media to define, express and convey product information in conception, design, and manufacture, engineering graphics play an extremely important role in technical and management work. Following the basic requirements of engineering graphics course teaching, configuration design is a teaching activity of conceiving and describing the plane figures, three-dimensional structure, part structure and component structure, etc. Through the configuration design training, it can deepen students' understanding and mastering the teaching contents, and further cultivate their ability to visualize innovative thinking. In this paper, the necessity of the teaching of configuration design in engineering graphics is analyzed, the basic ideas of the teaching of configuration design are introduced, and the main forms that can be adopted in the configuration design training are given, including configuration design based on geometric elements, shape configuration design based on one view, two views as well as three views and configuration design of chimeric shape based on given shape, etc.
\end{abstract}

Keywords: Engineering graphics, Configuration design, Innovative thinking, Drawing teaching

\section{INTRODUCTION}

The process of configuration design is the process of thinking in terms of images, through which people's imagination of space, conception of shape and expression of shape can be closely combined. The purpose of configuration design is to cultivate the ability of thinking and innovation and to train the engineering quality as well. Through the study and training of shape conception method, students' imagination of space and thinking ability can be developed, their drawing ability and reading ability can be improved, and then their visual innovative thinking and innovative design ability can be enhanced, which are conducive to excavating students' creative potential. Configuration design is the basis of industrial product design. Through the training of configuration design, students' awareness of engineering design and engineering innovation can be quickly established and their engineering quality can be gradually improved.

\section{THE NECESSITY OF CONFIGURATION DESIGN TEACHING}

Engineering graphics is a subject that studies the expression, communication and transmission of engineering and product information. Engineering graphics is the carrier of engineering and product information, and the language of expression and communication in engineering circles. One of the basic tasks of engineering graphics teaching is to cultivate students' creative configuration design ability, and one of the basic contents of engineering graphics is the fundamentals of configuration method. Configuration design training is a practical and feasible method to cultivate innovative ability. It is also a good carrier to cultivate students' graphic thinking ability. The ability of configuration design is an indispensable part of the cultivation of the innovation ability of students majoring in engineering.

\section{THE TEACHING THOUGHT OF CONFIGURATION DESIGN}

Configuration design ability is mainly the comprehensive ability of image thinking, conceptual expression and innovative design, which reflects the ability of engineering and technical personnel to build the corresponding shape structure according to the functional requirements of a product. Configuration design ability is the basis of innovative design. In classroom teaching, there are different forms of configuration design training methods for different teaching contents. The main thought is to take the configuration design of shape as the main line throughout the whole teaching process and select typical examples to conduct configuration design training.

Firstly, sketching is an effective means of configuration design. Creative configuration design first needs to record the designer's ideas in time, and the method of recording is to draw sketches or axonometric drawings freehand. Through the training of surveying and mapping of typical objects, the teaching in this field can be well completed and the ability of freehand sketching can be effectively 
trained, so that students can express their design ideas through sketches.

Secondly, pay attention to cultivating students' consciousness of three-dimensional thinking. The method of configuration design can restore the three-dimensional world. At present, the production process of many products in the mechanical manufacturing industry is carried out around three-dimensional modeling. The previous process of conceiving three-dimensional entities from two-dimensional plane graphics has become gradually weakened. Therefore, in the teaching process, cultivating students' awareness of three-dimensional thinking must be stressed. This requires that in addition to the traditional physical model in teaching, such teaching aids as multimedia and three-dimensional teaching software, etc. should be appropriately used to strengthen the training of students' observation ability and increase the accumulation and reserve of a variety of shapes.

Thirdly, make full use of computer assisted instruction. With the development of modern science and technology, the hardware construction and software assurance of computer assisted instruction provide great support for the teaching of configuration design. On the one hand, the teaching of configuration design is carried out in the classroom or training room, on the other hand, the purpose of space configuration can be achieved by using computer software.

\section{THE MAIN MODES OF CONFIGURATION DESIGN TRAINING}

\subsection{Plane Graphic Configuration Design}

Although the outline shape of space objects is different, it can be regarded as being composed of various geometric graphics. The commonly used geometric drawing method and plane drawing method are the basis of engineering drawing and mechanical drawing, which must follow the relevant provisions of national standards when drawing plane graphics. Therefore, when the content of configuration design is integrated into teaching, the content of national standards cannot be changed. Configuration design mainly aims at the characteristic shape of plane outline under which students are required to design plane graphics and mark dimensions with certain creative constraints. For example, design the plane contour graphics of a common object in everyday life, the contour graphics should contain a known-line, middle-line and connecting-line (a straight line or arc line) and design the relevant size (see Fig.1).

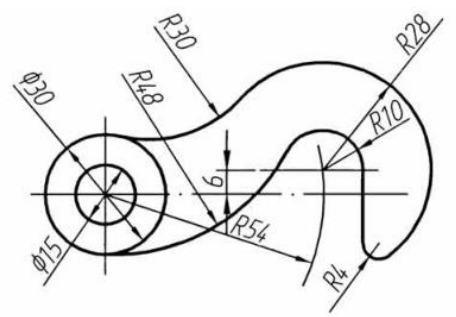

Figure 1 Plane graphic configuration design

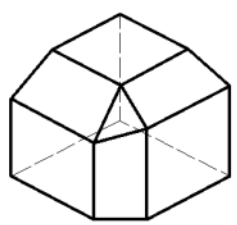

(a)

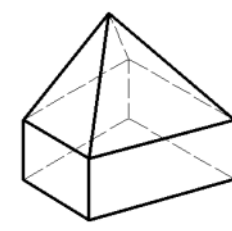

(b)
Figure 2 Geometrical element configuration design

\subsection{Geometric Element Configuration Design}

Geometric elements are mainly points, lines and planes. Students are required to design three-dimensional configurations including various positions of straight lines and planes. For example, they are required to design a solid whose surface contains vertical lines, parallel lines and general position lines of the projection plane (Fig.2a); or they are required to design a solid whose surface contains the vertical plane, the parallel plane and the general position plane of the projection plane (Fig.2b).

\subsection{Configuration Design of Combination}

When teaching the three views and axonometric drawings of combination, the three-dimensional model has been introduced into the teaching in an all-round way, and the focus is to cultivate students' ability to express three-dimensional space shape with two-dimensional plane graphics. The teaching objectives requires students to master the projection characteristics and drawing methods of stereoscopic projection, grasp the method of 3D shape conception, design and innovation and be familiar with the use of shape analysis and line analysis to draw and read the projection of the combination of shapes, and the mark the size of the combination. In class, under the functional needs of shape, students can be guided to imagine and conceive combinations according to known views, create designs and draw other views and axonometric drawings corresponding to the projection relationship of known views, and compare them with each other to identify the right and wrong. When raising questions, students thinking should be developed in an all-round way from easy to difficult, step by step. In teaching, design the configuration design training questions from the following aspects. 


\subsection{Design Different Combinations with a Given View}

As shown in Fig.3, according to the main view, design a combination of different shapes and draw the left view and axonometric drawing. Through the conception of drawing, identify that one view cannot clearly express the spatial shape.

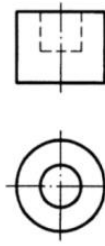

(a)
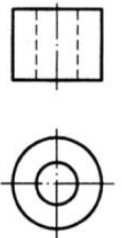

(b)
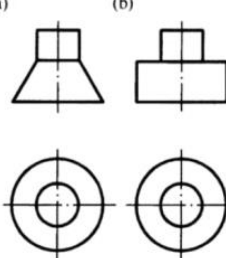

(f)

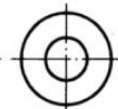

(g)
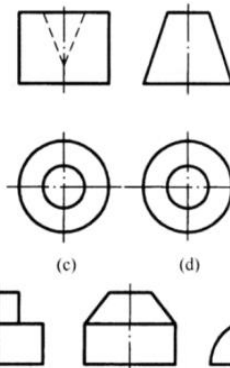

(d)

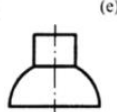

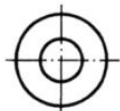

(h)
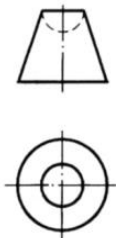

Figure 3 Design different combinations with given views

\subsection{Design Different Combinations with Two Given Views}

As shown in Fig.4, conceive and design the combination of different shapes according to the main and top views and draw the left view and axonometric drawing. Through drawing, remind the students that the two views sometimes cannot express the shape clearly.

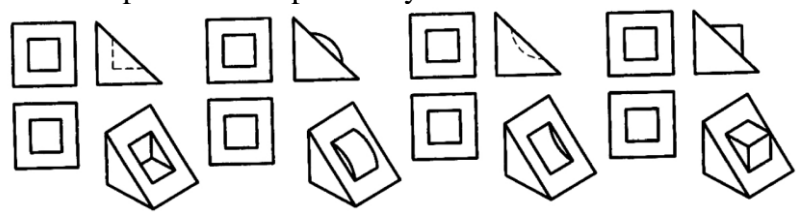

Figure 4 Design different combinations with given main views and top views

\subsection{Design Different Combinations with Three Given Views}

As shown in Fig.5, it is still possible to conceive and design combinations of different shapes according to the main, top and left views. In this example, it can either cut 8 corners (Fig.5a) or 7 corners (Fig.5b) on the cube. Through conceptual drawing, it is found that different spatial shapes can be designed, thus reminding students that three views are not sufficient enough to express the shape of objects uniquely in some cases.

\subsection{Conceive and Design a Combination with Which It Is Embedded with Three Given Views}

For example, Fig.6a represents a spatial combination, then how can we configure and design another combination that fits into it? Remind the students that two combinations mutually embed may constitute a cylinder. At this time they can conceive and imagine drawing the combination shown in Fig.6b. Students can also give full play to their imagination and conceive other objects. In such training, students' spatial imagination can be fully explored.

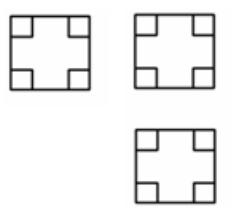

(a)

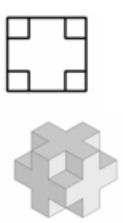

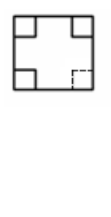

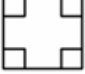

(b)
Figure 5 Design combinations of different shapes with given three views
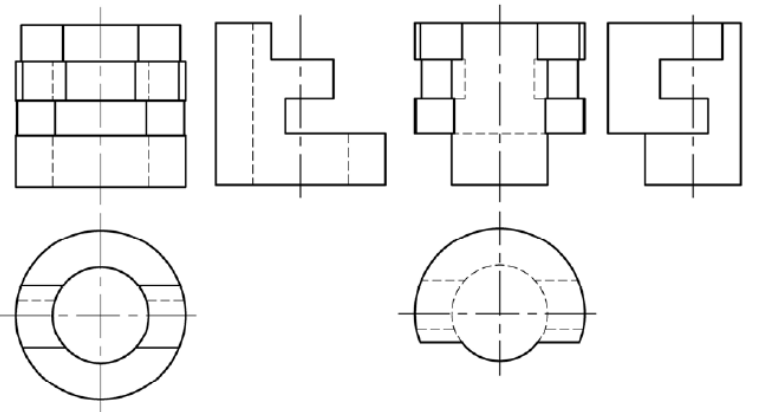

(a)

(b)

Figure 6 Mutually embedded combinations

\section{Conclusion}

The configuration design and training in engineering drawing course design various training examples, which is accomplished by different teaching methods based on different teaching contents. Strengthening the training of students' configuration design is very helpful to cultivate students' spatial imagination and spatial conception ability, and is an important way to improve students' innovative thinking ability. At the same time, the teaching objectives are intended to cultivate students' ability to use drawing software to draw engineering drawings and to design three-dimensional modeling. In the course of strengthening the students' configuration design training, the teaching should rely more on computer graphics software and solid modeling software. Through computer technology, three-dimensional solid model can be established in three-dimensional space, and the solid model can be observed from different perspectives by changing different viewpoints, so as to better complete the configuration innovation design. 


\section{REFERENCES}

[1] Dong Guoyao. Theory on engineering graphics configuration design. Journal of higher education research on mechanical industry, 1993, 38(2) (in Chinese)

[2] Chen Xianghe. The research on view structure analysis and its space configuration method [J]. Journal of Engineering Graphics, 2006(3). (in Chinese)

[3] Tong Bingshu. The cultivation of student's innovation ability in teaching of engineering graphics $[\mathrm{J}]$. Journal of engineering graphics, 2008(6). (in Chinese)

[4] Lin Jinzhu. Strengthen the combination of configuration design with training creative thinking ability [J]. Journal of Chuzhou vocational and technical college, 2009, 6(3). (in Chinese)

[5] Liu Jinying. The research on configuration design in the teaching of engineering graphics course[J]. Science and Technology Information, 2010(9). (in Chinese)

[6] Sun Hui. The exploration and practice on strengthening configuration innovation design ability in engineering graphics education [J]. Journal of Donghua University, 2010(4). (in Chinese) 